\title{
Impact of video quality when evaluating video-assisted cardiopulmonary resuscitation: a randomized, controlled simulation trial
}

Christopher Plata ${ }^{1,2}$, Martin Nellessen ${ }^{1}$, Rebecca Roth ${ }^{3}$, Hannes Ecker ${ }^{1}$, Bernd W. Böttiger ${ }^{1}$, Johannes Löser ${ }^{4}$ and Wolfgang A. Wetsch ${ }^{1 *}$ (D)

\begin{abstract}
Background: Although not routinely established during cardiopulmonary resuscitation (CPR), video-assisted CPR has been described as beneficial in the communication with emergency medical service (EMS) authorities in out-ofhospital cardiac arrest scenarios. Since the influence of video quality has not been investigated systematically and due to variation of quality of a live-stream video during video-assisted CPR, we investigated the influence of different video quality levels during the evaluation of CPR performance in video sequences.

Methods: Seven video sequences of CPR performance were recorded in high quality and artificially reduced to medium and low quality afterwards. Video sequences showed either correct CPR performance or one of six typical errors: too low and too high compression rate, superficial and increased compression depth, wrong hand position and incomplete release. Video sequences were randomly assigned to the different quality levels. During the randomised and double-blinded evaluation process, 46 paramedics and 47 emergency physicians evaluated seven video sequences of CPR performance in different quality levels (high, medium and low resolution).

Results: Of 650 video sequences, CPR performance was evaluable in 98.2\%. CPR performance was correctly evaluated in $71.5 \%$ at low quality, in $76.8 \%$ at medium quality, and in $77.3 \%$ at high quality level, showing no significant differences depending on video quality $(p=0.306)$. In the subgroup analysis, correct classification of increased compression depth showed significant differences depending on video quality $(p=0.006)$. Further, there were significant differences in correct CPR classification depending on the presented error $(p<0.001)$. Allegedly errors, that were not shown in the video sequence, were classified in $28.3 \%$, insignificantly depending on video quality. Correct evaluation did not show significant interprofessional differences $(p=0.468)$.

Conclusion: Video quality has no significant impact on the evaluation of CPR in a video sequence. Even low video quality leads to an acceptable rate of correct evaluation of CPR performance. There is a significant difference in evaluation of CPR performance depending on the presented error in a video sequence.
\end{abstract}

\footnotetext{
* Correspondence: wolfgang.wetsch@uk-koeln.de

'University of Cologne, Faculty of Medicine and University Hospital of Cologne, Department of Anaesthesiology and Intensive Care Medicine, Kerpener Strasse 62, 50937 Cologne, Germany

Full list of author information is available at the end of the article
}

(c) The Author(s). 2021 Open Access This article is licensed under a Creative Commons Attribution 4.0 International License, which permits use, sharing, adaptation, distribution and reproduction in any medium or format, as long as you give appropriate credit to the original author(s) and the source, provide a link to the Creative Commons licence, and indicate if changes were made. The images or other third party material in this article are included in the article's Creative Commons licence, unless indicated otherwise in a credit line to the material. If material is not included in the article's Creative Commons licence and your intended use is not permitted by statutory regulation or exceeds the permitted use, you will need to obtain permission directly from the copyright holder. To view a copy of this licence, visit http://creativecommons.org/licenses/by/4.0/. The Creative Commons Public Domain Dedication waiver (http://creativecommons.org/publicdomain/zero/1.0/) applies to the data made available in this article, unless otherwise stated in a credit line to the data. 
Trial registration: German Clinical Trial Register (Registration number DRKS00015297) Registered on 2018-08-21.

Keywords: Cardiopulmonary resuscitation, CPR, Video-assisted CPR, V-CPR, OHCA

\section{Background}

Sudden cardiac arrest is the most immediate lifethreatening medical condition. Despite all advances in prevention and treatment, it accounts for approximately half of all deaths from cardiovascular disease [1], and is hence among the leading causes of death in industrialised nations [2]. Brain tissue is very vulnerable to hypoxaemia, and irreversible damage starts to occur 3 to 5 min after the onset of circulatory arrest. Unfortunately, despite rapid response by Emergency Medical Services (EMS), professional helpers often arrive too late on scene to save patients' lives or avoid irreversal neurological damage. Early initiation of cardiopulmonary resuscitation (CPR) by bystanders can bridge the gap till the arrival of medical professionals and hence could help save hundreds of lives every day [3, 4]. Sadly, in most countries, bystander CPR in out-of-hospital cardiac arrest is performed in only $15-50 \%$ [5], although witnessed in more than $60 \%$ of the cases $[2,6]$.

By introducing dispatcher-assisted telephone CPR (TCPR), which contains structured instruction on how to perform CPR, the probability of surviving cardiac arrest has considerably increased [4, 7-10]. However, instructing bystanders, who are mostly unexperienced in providing CPR, remains challenging. Due to the limitations of the audio-only communication, dispatchers cannot see what the bystander does, and hence cannot evaluate CPR quality or give corrective feedback.

Despite technical inventions and revolutionary advantages over the last decades, like the ubiquitous coverage of high-speed internet connectivity and mobile multifunctional smartphones, the predominant way of communication with emergency authorities has remained the traditional audio contact. Video-assisted CPR (V$\mathrm{CPR}$ ) is a new possible way of instructing bystanders during CPR [11, 12]. Although first studies of videoassisted CPR started over a decade ago, video-guided CPR did not prove to be superior to conventional CPR at those days. To date, video-assisted CPR remains almost unknown in EMS systems around the world. Recently, this feature has been implemented in a software that can be routinely used for EMS dispatch centres, and first studies have shown that this technology can be used to judge a layperson's CPR efforts and correct them, if necessary [13].

However, to date there is no priority for mobile data used for emergency calls, hence livestream video quality can vary due to reduced data transfer rates or bad mobile internet coverage. The necessary quality of a video in order to allow accurate judgement of CPR efforts has not been studied so far. Furthermore, in published research, there is heterogeneity concerning the qualification of the evaluating person.

The aim of this study was to determine the necessary video quality in order to be able to evaluate CPR quality. In addition, we wanted to investigate whether there is an influence of the evaluator's profession on evaluation of CPR quality in a video sequence.

\section{Methods \\ Study design}

This prospective, randomized, controlled, parallel group and double-blind simulation trial was conducted in the University Hospital of Cologne and the EMS Department of the Fire Brigade of Kerpen, Germany. The study was approved by the Ethics Committee of the University Hospital Cologne (Approval number 18-130, 12th of February 2019) and was registered at the German Clinical Trial Register (Registration number DRKS00015297). Data was collected from September 2019 to February 2020. Written and informed consent was obtained from each participant prior to inclusion.

\section{Materials}

CPR was simulated using a standardized CPR training manikin (Ambu Man basic, Ambu GmbH, Bad Nauheim, Germany). Video sequences were recorded with a Tamron objective (Tamron SP 24-70 mm F/2.8, Tamron Corporation, Saitama, Japan) on a Nikon D750 (Nikon Corporation, Tokyo, Japan) with a resolution of $1920 \times$ 1080 pixels per inch and 30 frames per second, to obtain a video with maximum quality. The camera was placed laterally of the manikin on a tripod of $1.80 \mathrm{~m}$ height, facing the manikin and the helper performing CPR from approximately $2.5 \mathrm{~m}$ of distance. A metronome (metronom beats app, Stonekick, London, UK; downloaded from Google Play Store) was set to $110 \mathrm{bpm}$ (or the corresponding lower/higher frequency) to ensure guidelineconformant compression frequency, and the internal compression depth indicator of the manikin was used to ensure sufficient compression depth. In the final video sequences, neither metronome nor compression depth indicator was visible respectively audible. Seven high quality video clips of $12 \mathrm{~s}$ each were recorded, showing either guideline-conformant optimum performance compression-only CPR, or a typical error often made during CPR (Table 1). 
Table 1 CPR parameters and errors shown in the video sequences

\begin{tabular}{|c|c|c|}
\hline 1 & Correct CPR & $\begin{array}{l}\text { Compression depth } 5-6 \mathrm{~cm} \text {, compression rate } 110 / \mathrm{min} \text {, } \\
\text { correct hand position, complete thorax release after compression }\end{array}$ \\
\hline 2 & Low compression rate & $80 \mathrm{~min}^{-1}$, others correct \\
\hline 3 & High compression rate & $140 \mathrm{~min}^{-1}$, others correct \\
\hline 4 & Increased compression depth & $>70 \mathrm{~mm}$, others correct \\
\hline 5 & Superficial compression depth & 30-40 mm, others correct \\
\hline 6 & Wrong hand position & Epigastric compression point, others correct \\
\hline 7 & Incomplete thorax release & Remaining depth > $20 \mathrm{~mm}$, others correct \\
\hline
\end{tabular}

Video resolution was artificially reduced by using a software tool (XMedia Recode, V. 3.4.3.6, Eschenbergen, Germany) to obtain three quality levels: high (resolution 1920x1080ppi), medium (resolution 320x240ppi) and low (resolution $128 \times 96 \mathrm{ppi}$ ), all with a frame rate of $30 \mathrm{fps}$ (Fig. 1). High quality served as standard, while the lower resolutions are typically used on mobile devices, when data is transferred via wireless networks and compression is necessary. Video sequences and quality levels were randomized with a randomization algorithm provided by the Institute of Medical Statistics and Computational Biology (IMSB) of the University of Cologne. During the evaluation phase, video sequences were analyzed on an ASUS X541UA laptop with a 15.6" screen. A standardized questionnaire with multiplechoice answers was used to evaluate CPR quality.

\section{Study protocol}

Foty-six paramedics and 47 emergency physicians were randomly recruited on the campus of the University Hospital of Cologne, Germany and the EMS Department of the Fire Brigade of Kerpen, Germany. There were no primary exclusion criteria.

Each evaluator had to judge seven video sequences (Fig. 2). Six of those included the six different error types and one did not comprise any error. Only one error was

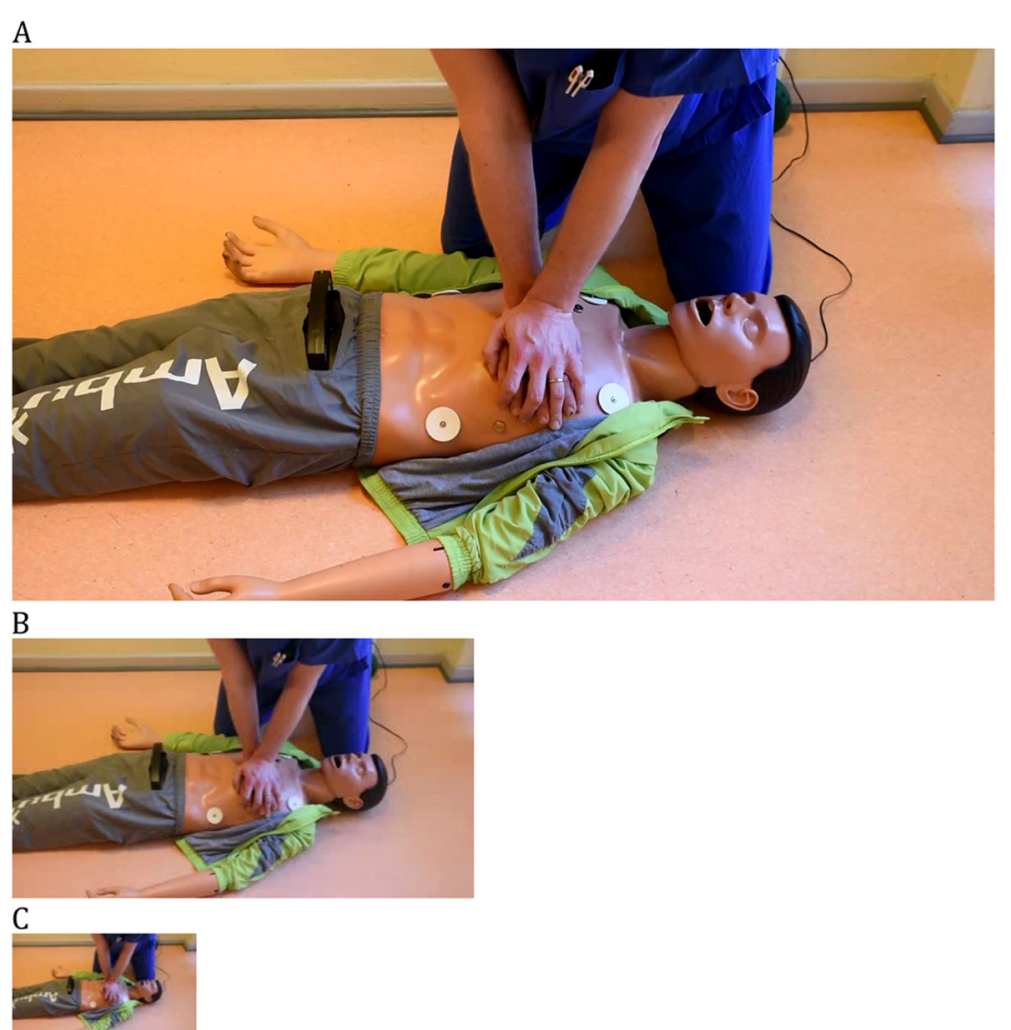

Fig. 1 Still frames of the videos used for evaluation: A high resolution (1920x1080ppi), B medium resolution (320x240ppi) and C low resolution (128x96ppi) 


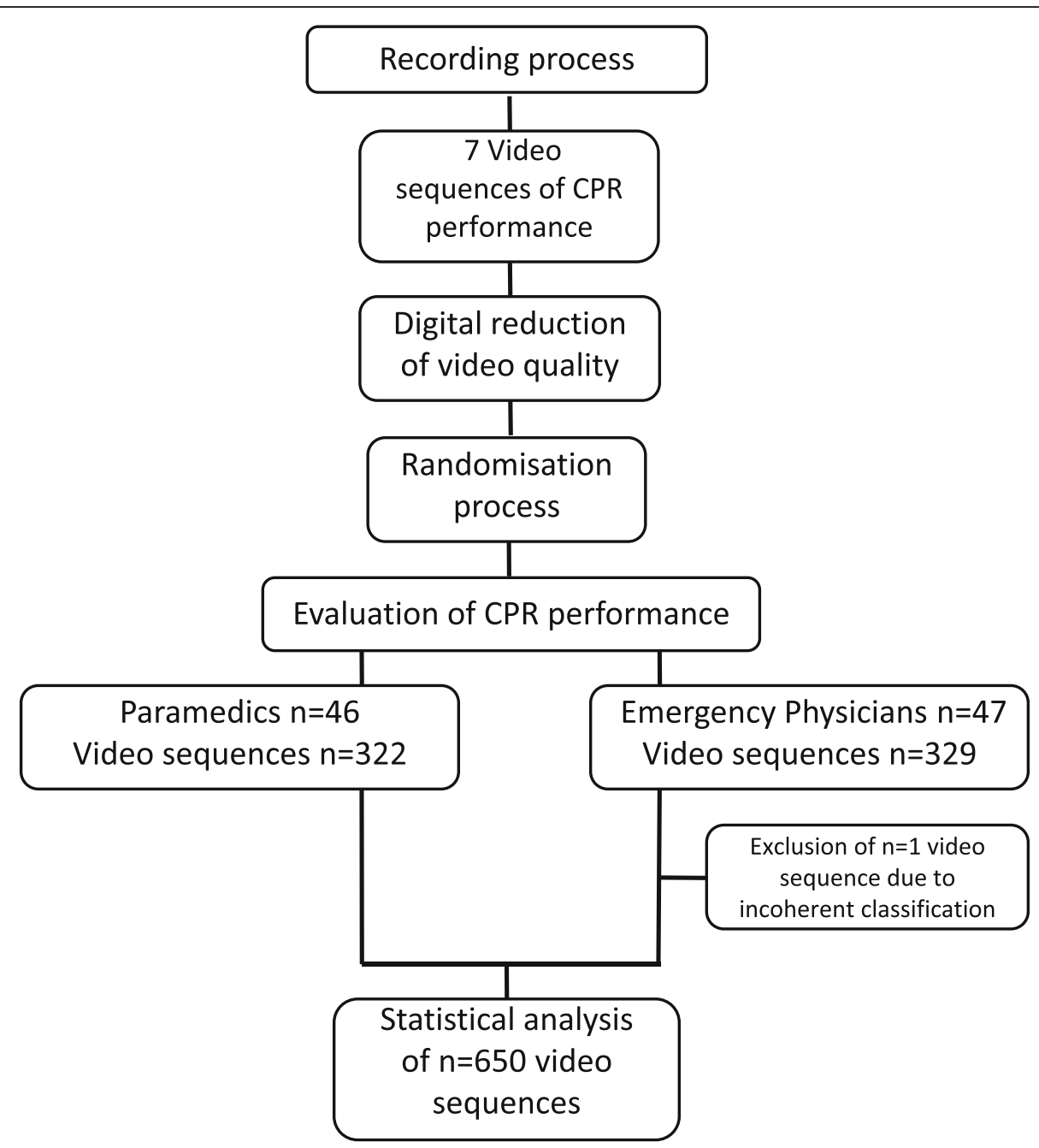

Fig. 2 Study flow chart. During the recording process, seven video sequences of CPR were recorded. After reduction of the video quality, video sequences were randomly assigned to the different quality levels and evaluated by either paramedics or emergency physicians, CPR: cardiopulmonary resuscitation

included per video sequence (see Table 1). Participants were asked to evaluate CPR performance after each video sequence, using a standardized questionnaire with multiple-choice answers about compression rate, compression depth, compression point and release after compression. Additionally, participants were not informed about the number of errors in one video sequence and, thus, indication of more than one error was possible. During the evaluation of every video sequence, participantshad the possibility to indicate each category as "not evaluable". There was no time limit for answering the questionnaire.

\section{Measurements and outcomes}

The primary outcome parameter was correct identification of the error presented in the video sequence depending on the video quality. Secondary outcome parameters consisted of classification of CPR performance depending on the presented error, false classification of correct CPR performance, frequencies of "CPR performance not evaluable", indication of further, allegedly errors and finally differences in classification depending on different professions.

\section{Statistical analysis}

The required sample size was calculated in order to achieve $80 \%$ power at a significance level of $5 \%$ to detect a minimal difference in the percentage of error recognition between two different qualities of $25 \%$, i.e. assuming a detection rate of 95 vs. $70 \%$. Sample size calculation was accounted for a) the dependency of the seven ratings conducted by the same evaluator by assuming an intraclass correlation coefficient of 0.8 and b) a drop-out rate of $10 \%$. When recruiting a total of 100 evaluators, the actual power of a chi-square test to detect a difference in proportions of $25 \%$ was estimated as $81 \%$ (SAS 
9.3, Cary, North Carolina, USA). For each evaluator separately, error types and qualities were randomly assigned to video sequences, such that each evaluator had to judge each quality at least twice. For random assignment of error types and qualities, the software "R" (The R Foundation for Statistical Computing. Vienna, Austria) was employed. Descriptive analyses present numbers and percentages for categorical variables. For statistical analyses, categories out of the seven CPR performances were formed: low and high compression rate were summarized in the category "compression rate", low and increased compression depth in the category "compression depth" and finally hand position and thorax release. Where exact Fisher's test could not be calculated due to limited computational capacities, Chi-square tests were applied to investigate associations between categorical variables. Missing data were not imputed. As this is an exploratory study, no correction for multiple testing was performed. $P$ values $<0.05$ were regarded as statistically significant. Statistical computations were carried out using IBM SPSS Statistics version 25 .

\section{Results}

In total, 651 video sequences were presented to the evaluators, including 322 video sequences being evaluated by 46 paramedics and 329 video sequences analyzed by 47 emergency physicians (Fig. 2). All questionnaires were returned. One volunteer of the emergency physicians-group gave inconsistent answers regarding release after compression in a video sequence of medium quality, showing correct CPR performance. Thus, 650 answers (99.8\%) were included for statistical analysis.

\section{Classification of CPR performance depending on video quality}

Errors presented in the video sequences were evaluated correctly in $71.5 \%$ at low quality, in $76.8 \%$ at medium quality and in $77.3 \%$ at high quality (Fig. 3). Overall, $75.2 \%$ of all presented video sequences were correctly evaluated. There was no significant difference in correct error classification depending on quality levels of the presented video sequences $(p=0.306)$.

Regarding the classification of the specific errors depending on the different video quality levels, results are shown in Fig. 4. Wrong hand position was recognized correctly in $100 \%$ at low and medium quality levels and in $97 \%$ at high quality level, revealing no significant differences in dependence of video quality $(p=1.0)$. Likewise, correct CPR performance $(p=0.363)$, low and high compression rate $(p=0.617 ; p=0.847)$, superficial compression depth $(p=0.619)$ and incomplete release $(p=$ 0.198 ) did not show significant differences depending on video quality. In contrast, $13.8 \%$ of increased chest compressions at low quality, $50 \%$ at medium quality and $42.9 \%$ at high quality level were classified correctly, revealing a significant difference in different video quality stages $(p=0.006)$.

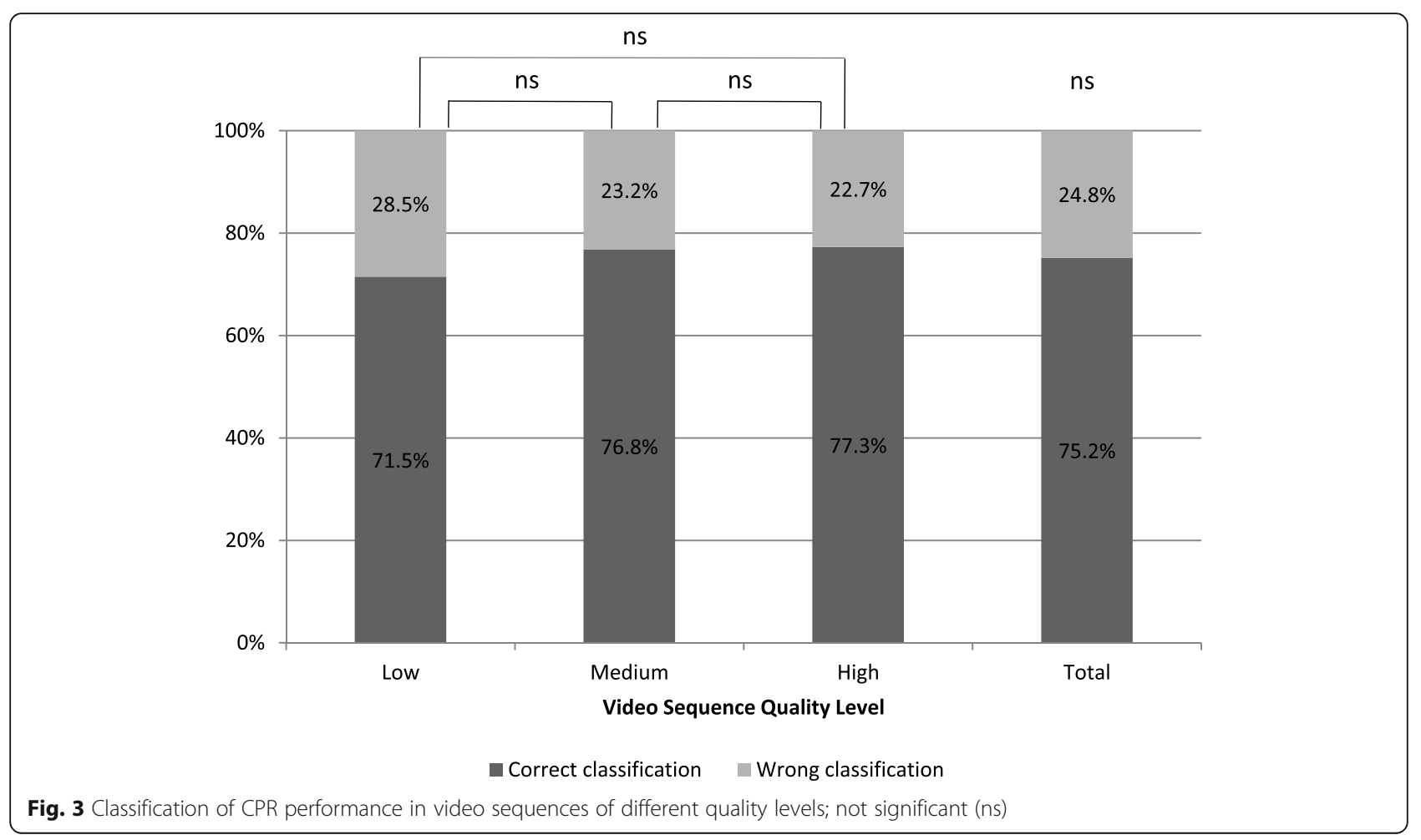




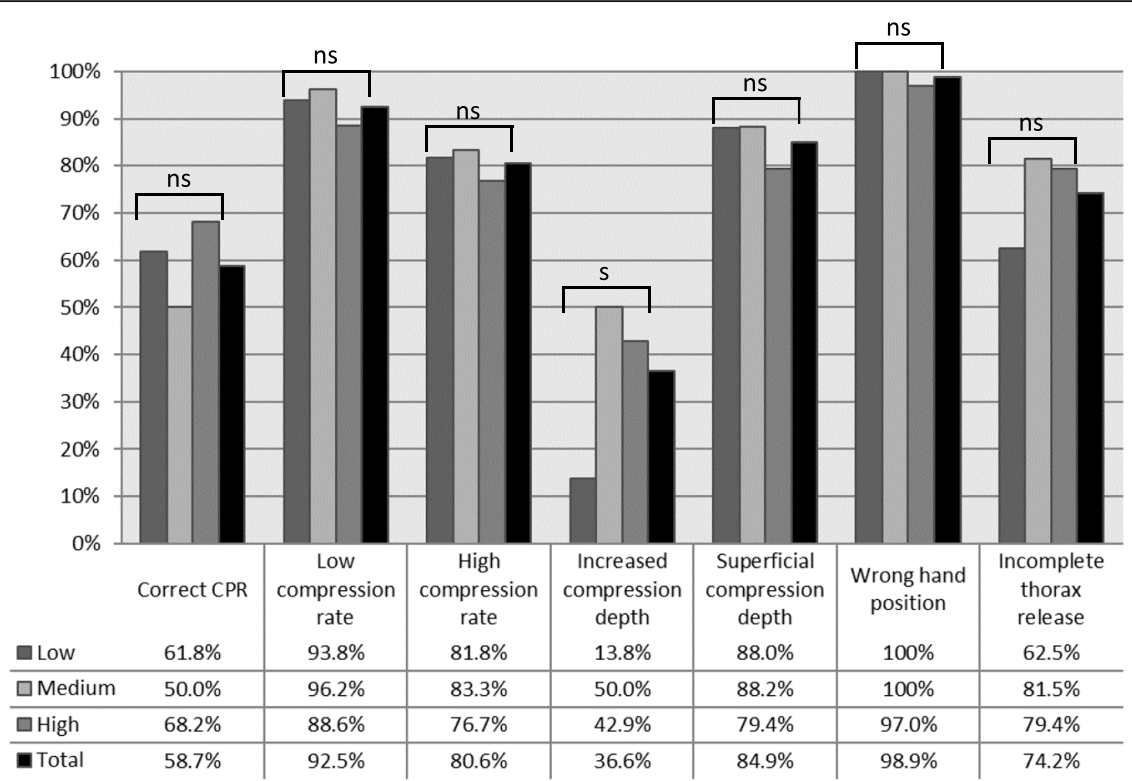

Fig. 4 Correct classification of errors during CPR depending on different video quality levels; not significant (ns), significant (s)

\section{Classification of CPR performance depending on the presented error}

Further analyses focussed on the frequencies of correct error classification depending on the presented error. Thereby, we found a significant difference in correct classification depending on the presented error $(p<$ 0.001; Fig. 5). In the following subgroup analysis, we evaluated differences in correct classification between two errors, as shown in Table 2.
Correct CPR was falsely evaluated as "incorrect" in $41.3 \%$ of all videos and thus evaluators attributed errors to the correct CPR performance. Correct CPR performance was falsely classified as low compression rate (21.6\%, [n=8]), superficial compression depth and incomplete thorax release (each $27 \%,[n=10]$ ), high compression rate, increased compression depth and wrong hand position (each $8.1 \%,[n=3]$ ).

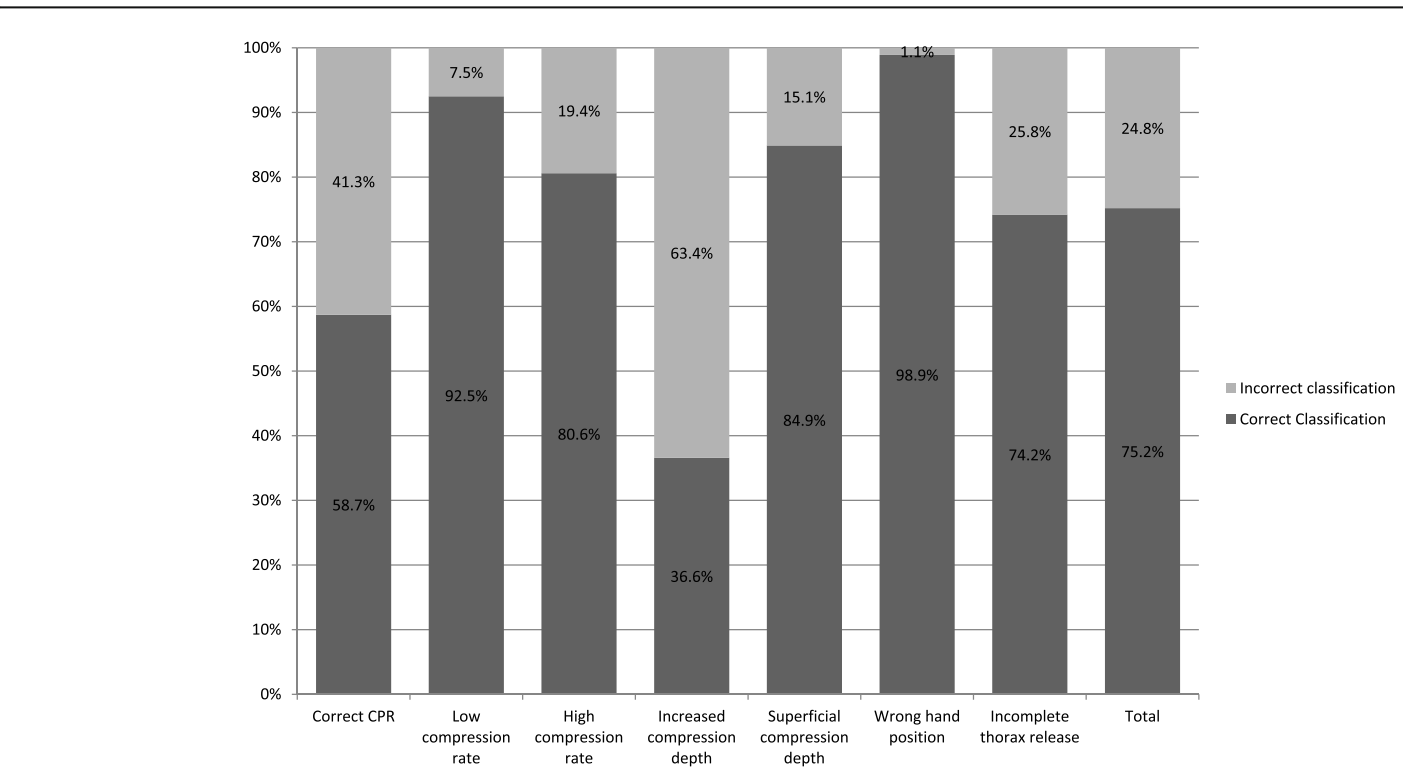

Fig. 5 Frequencies of correct error classification depending on the presented error over all quality stages $(p<0.001)$ 
Table 2 Pairwise analysis of CPR performances and levels of significance

\begin{tabular}{|c|c|c|c|}
\hline CPR performance & & CPR performance & $p$-value \\
\hline Correct CPR & vs & Low compression rate & $<0,001$ \\
\hline Correct CPR & vs & High compression rate & 0,001 \\
\hline Correct CPR & vs & Increased compression depth & 0,003 \\
\hline Correct CPR & vs & Superficial compression depth & $<0,001$ \\
\hline Correct CPR & vs & Wrong hand position & $<0,001$ \\
\hline Correct CPR & vs & Incomplete thorax release & 0,03 \\
\hline Low compression rate & vs & High compression rate & 0,03 \\
\hline Low compression rate & vs & Increased compression depth & $<0,001$ \\
\hline Low compression rate & vs & Superficial compression depth & 0,163 \\
\hline Low compression rate & vs & Wrong hand position & 0,064 \\
\hline Low compression rate & vs & Incomplete thorax release & 0,001 \\
\hline High compression rate & vs & Increased compression depth & $<0,001$ \\
\hline High compression rate & vs & Superficial compression depth & 0,561 \\
\hline High compression rate & vs & Wrong hand position & $<0,001$ \\
\hline High compression rate & vs & Incomplete thorax release & 0,381 \\
\hline Increased compression depth & vs & Superficial compression depth & $<0,001$ \\
\hline Increased compression depth & vs & Wrong hand position & $<0,001$ \\
\hline Increased compression depth & vs & Incomplete thorax release & $<0,001$ \\
\hline Superficial compression depth & vs & Wrong hand position & 0,001 \\
\hline Superficial compression depth & vs & Incomplete thorax release & 0,101 \\
\hline Wrong hand position & vs & Incomplete thorax release & $<0,001$ \\
\hline
\end{tabular}

\section{CPR performance not evaluable}

In 12 of 650 video sequences (1.8\%), a CPR category (compression rate, compression depth, hand position, thorax release) was marked as "not evaluable". In case of low video quality, evaluators indicated a CPR category to be not evaluable in 6 video sequences $(0.9 \%), 1$ video sequence $(0.2 \%)$ in a medium quality level and in 5 high quality video sequences $(0.8 \%)$, revealing no significant difference depending on video quality level $(p=1.0)$.

\section{Indication of further, allegedly errors}

As shown in Table 1, there was only one error per video sequence (except for the video with correct CPR, which contained no error). However, in 184 of 650 video sequences (28.3\%), 262 additional errors, that were not shown in the video sequence, were indicated by the evaluators (Fig. 6b). While allegedly errors did not significantly depend on video quality levels $(p=0.422)$, the presented error was of significance for the indication of additional errors $(p<$ $0.001)$. Lowest frequency of additional allegedly errors was found in video sequences of correct CPR performance ( 7 of $92=7.6 \%$, Fig. 6a). Highest number of indicated additional allegedly errors was seen in video sequences showing wrong hand position during CPR
(43 of $93=46.2 \%$ ), revealing a significant difference compared to correct CPR $(p<0.001)$.

\section{Error classification by different professionals}

This study was not powered to find differences in the evaluation of different professions. However, our results reveal some interesting findings regarding interprofessional differences between paramedics and emergency physicians. Summarizing all quality stages, correct classification of the presented errors was achieved in $73.9 \%$ for paramedics and in $76.5 \%$ for emergency physicians, showing no significant interprofessional difference $(p=$ 0.468). Significant interprofessional differences were only seen in low quality video sequences, where $78.7 \%$ $(n=85)$ of the presented errors were correctly evaluated by emergency physicians, but only $64.2 \%(n=68)$ by paramedics $(p=0.023)$. In medium and high quality, interprofessional evaluation showed no significant differences ( $p=0.873$ and $p=0.419$ respectively). However, by analysing the results of emergency physicians and paramedics separately, there was a significant influence of the video quality level on correct errors classification by paramedics $(p=0.021)$ but not by emergency physicians $(p=0.8)$. For paramedics evaluating a video sequence, increased chest compressions in a low video quality were significantly less frequently recognized, 

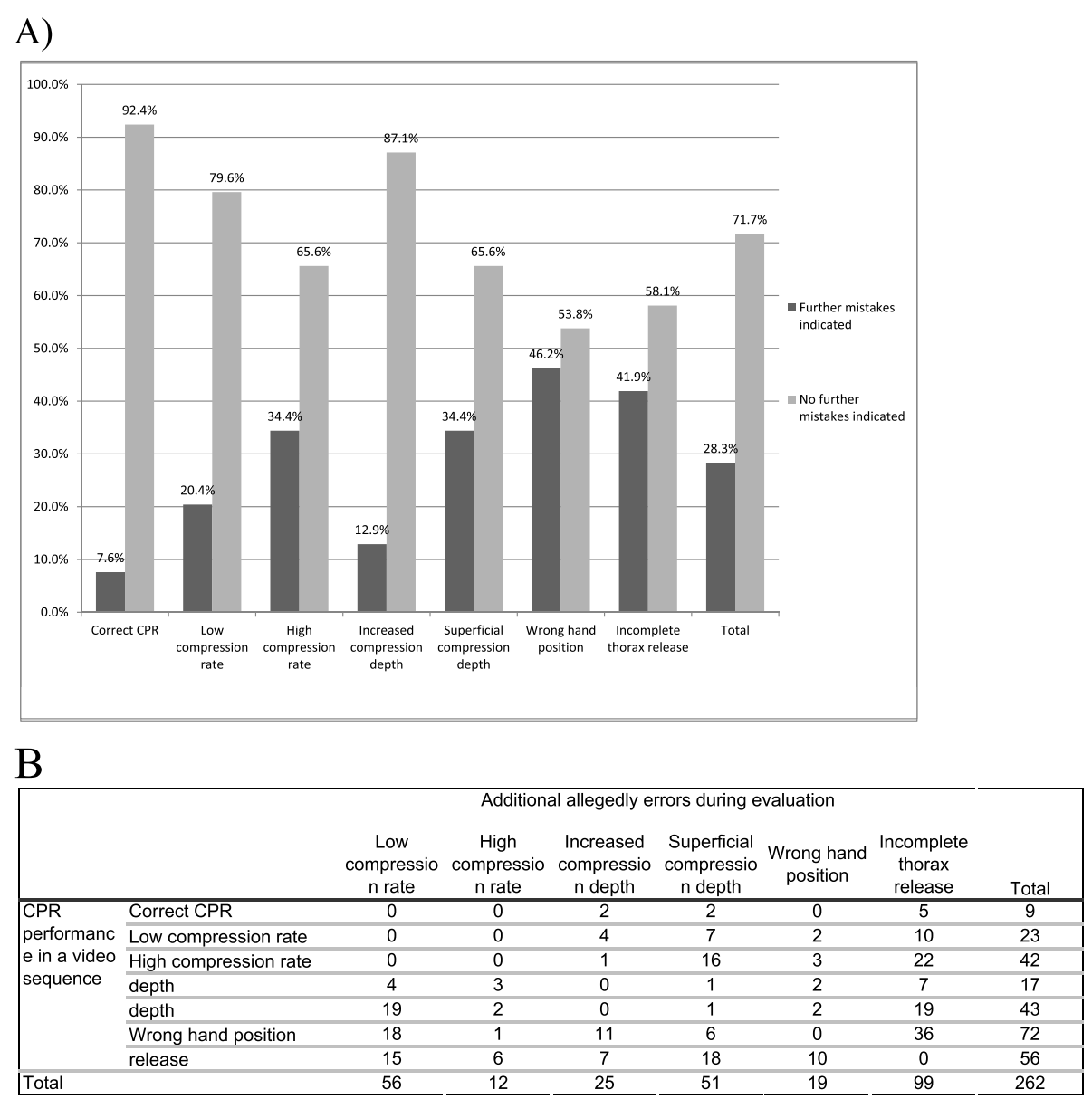

Fig. 6 A Frequencies of additional errors depending on the presented CPR performance in a video sequence $(p<0.001)$; B crosstabulation showing allocation and summation of all additional allegedly errors during evaluation of a video sequence (indication of more than one error was possible)

compared to medium and high quality levels $(12.5 \%$ vs. $60 \%$ vs. $60 \% ; p=0.007$ ). Further, video quality had a significant influence on detection of incomplete thorax release after compression evaluated by paramedics $(p=$ $0.045)$ but not by emergency physicians $(p=0.393)$. It was most frequently detected at a high quality level (92.3\%), followed by a medium quality level (73.3\%) and low quality level (50\%). In both professional groups, the presented error in a video sequence had a significant influence on correct error classification ( $\mathrm{p}_{\text {paramedics }}<$ 0.001; pemergency physicians $<0.001$ ). Finally, there was no interprofessional difference regarding indicating " $\mathrm{CPR}$ performance not evaluable" $(p=0.415)$ and indicating further errors $(p=0.728)$.

\section{Discussion}

The present study is the first to primarily focus on the influence of the video quality on the assessment of CPR effort during simulated V-CPR. Our results indicate that the assessment of CPR quality during video-assisted
CPR does not depend on the availability of high-quality video footage, since correct classification did not differ significantly by video quality.

Tremendous developments in technology have brought significant changes to daily life in regard to telecommunication over the last decades [14]. Most visibly during the 2020 SARS-Cov2 pandemic, telemedicine got a major boost and video-connections for doctor's appointments and hospital triage got more common $[15,16,17]$. Surprisingly, communication with emergency authorities - which is one of the most crucial communication imaginable - seems to be unaffected by these trends and remains a traditional "audio-only" phone call. So far, to our best knowledge, video-assisted CPR has only been adopted and is commercially available in very few regions [13, 18, 19]. Nevertheless, studies have demonstrated that video calls can provide additional information to improve emergency communication, especially in conjunction with CPR [11-13, 18-22]. 
However, comparing these studies is challenging, as they were mostly not standardized in regard to image quality used and often do not state any quality preferences at all. Further, the term "video-CPR" is not clearly defined and thus comprises not only video-assisted CPR. In a study by Bolle et al., conducted over a decade ago, the authors compared T-CPR and V-CPR with high school students using a Nokia N90 [20]. No superiority of the V-CPR group was shown, which was explained by the low video quality (video resolution $352 \times 288$ pixels, framerate $15 / \mathrm{s}$ ), leading to difficulties in identifying the details of CPR performance. Technical conditions in this study are comparable to our medium quality, however we used a framerate of $30 / \mathrm{s}$ which is more comparable to the state of the art of video-streaming. Another study by Stipulante et al. compared V-CPR to T-CPR on mobile phones with 180 students [23]. The authors showed that the use of a V-CPR protocol allowed the CPR to reach compression rates and depths close to guidelines and to reduce 'hands-off' events. However, device and image quality parameters were not mentioned. More recently, in two of our own previous studies, we tested VCPR via smartphones in a randomized controlled trial on 150 participants in a wifi-setting and under realistic conditions in a metropolitan area using a mobile telephone network $[13,18]$. Both studies used a video livestream from a Samsung Galaxy S7, which delivers a video quality comparable to the medium and high quality setting in the present study. However, video quality was not explicitly stated in these studies. Despite results showing an overall improvement of the CPR quality, authors emphasized that connection problems due to technical issues and signal degradation affected video quality, especially in the second study using the mobile internet coverage.

All of our video sequences were taped with a framerate of $30 \mathrm{fps}$, as lower frame rates may contribute to an aliasing phenomenon. We consciously did not evaluate video sequences with reduced framerates, as it is very unlikely that they will play an important part in the future with the introduction of $4 \mathrm{G}$ and $5 \mathrm{G}$ mobile data networks. Furthermore, it is quite possible that higher resolutions and framerates will be available also on mobile devices, as $4 \mathrm{k}$ resolution $(4096 \times 2160$ pixels $)$ and framerates up to $60 \mathrm{fps}$ become available, albeit not routinely implemented in video calls yet. Although this study did not show appropriate level of statistical power and sensitivity in regard to the interprofessional differences, results do not show a significant interprofessional difference in the evaluation of V-CPR. It can be carefully suggested that a variety of medical emergency professions can serve as dispatchers for V-CPR, since both professional groups identified over $70 \%$ of the CPR performances correctly. Furthermore, it must be kept in mind that our evaluators were confronted with videoassisted CPR for the very first time. None of them had any training with this technology. It can be hypothesized that any training may lead to further improved evaluation of V-CPR [23].

Furthermore, data in both simulated and actual patients have shown that even experienced clinicians are not very good at determining whether compressions are being one properly - this applies for "remote" observers as well as for those that are physically present at the time [24-27]. Judging other persons performing CPR is not taught in any course, and it seems as if those who need to judge other persons' CPR performance (e.g., 911 dispatchers or telemedicine paramaedics/doctors) might need special training towards evaluation of CPR quality. Further studies evaluating this finding are needed to gain insight into this phenomenon.

\section{Limitations}

This study was conducted with a resuscitation mannequin made for education and evaluation of medical personal, which is only a substitute for real patients. In accordance with the current European Resuscitation Council (ERC) Guidelines [28], we confined to a compression-only CPR approach. Future studies might incorporate ventilation in their CPR scenario. This study only investigated a maximum resolution of $1920 \times 1080$ pixels and framerate of $30 \mathrm{fps}$. It is quite possible that a higher resolution and framerate can lead to different results as $4 \mathrm{k}$ resolution $(4096 \times 2160$ pixels $)$ and framerates till $60 \mathrm{fps}$ are available. Videotaping was performed in a closed environment with sufficient light, constant weather, no disturbances and perfect technical facilities (e.g. tripod). Further evaluation in real-life situations is recommended. It must also be considered that we only used one video position. Different positions may positively or negatively influence the ability to recognize and correct certain mistakes.

\section{Conclusion}

Results of this study show that video quality has no significant impact on the evaluation of CPR in a video sequence. In fact, even low video quality leads to an acceptable evaluation of CPR performance. Except of the parameter "increased compression depth", not video quality but the presented error was of significance for correct CPR classification.

\section{Abbreviations}

ALS: Advanced Life Support; CPR: Cardio-pulmonary resuscitation; EMS: Emergency medical services; ERC: European Resuscitation Council; GRC: German Resuscitation Council; IBM: International Business Machines Corporation; ILCOR: International Liaison Committee on Resuscitation; IMSB: Institute of Medical Statistics and Computational Biology; OHCA: Outof-hospital cardiac arrest; SARS-CoV2 : severe acute respiratory syndrome 
coronavirus type 2; SAS: Statistical Analysis Systems; SPSS: Statistical Package for the Social Sciences; T-CPR: Telephone-assisted cardio-pulmonary resuscitation; V-CPR: Video-assisted cardio-pulmonary resuscitation

\section{Acknowledgments}

We would like to thank the Fire Brigade of the City of Kerpen, North RhineWestphalia, especially to Mr. Marco Lang and Mr. Marcus Arndt, for their help and support during study conduction. We would also like to thank all volunteers from this fire brigade for participating in our study.

This study was supported by the ERC Research NET.

\section{Availibility of data and materials}

The datasets used and/or analysed during the current study are available from the corresponding author on reasonable request.

\section{Authors' contributions}

CP and WAW had the idea and concepted the study; CP and WAW wrote the study protocol. CP, WAW and MN made the videos. CP an MN conducted the experiments with the participants. RR did the statistical analysis. CP, HE and WAW drafted the manuscript, BWB and JL critically revised it. All authors have read and approved the final manuscript.

\section{Funding}

This study was solely funded by institutional funding. Open Access funding enabled and organized by Projekt DEAL.

\section{Declarations}

\section{Ethics approval and consent to participate}

The study was approved by the Ethics Committee of the University Hospital Cologne (Approval number 18-130, 12th of February 2019) and was registered at the German Clinical Trial Register on August 21st, 2018 (Registration number DRKS00015297; URL https://www.drks.de/drks_web/ navigate.do?navigationld=trial. HTML\&TRIAL_ID=DRKS00015297). Written and informed consent was obtained from each participant prior to inclusion.

\section{Consent for publication}

\section{Not applicable.}

\section{Competing interests}

Bernd W. Böttiger is European Resuscitation Council (ERC) Treasurer and immediate past Board Director Science and Research; Chairman of the German Resuscitation Council (GRC); Member of the "Advanced Life Support (ALS) Task Force of the International Liaison Committee on Resuscitation (ILCOR); Member of the executive committee of the German Interdisciplinary Association for Intensive and Emergency Medicine (DIVI); Associated Editor of the European Journal of Anaesthesiology (EJA), Co-Editor of "Resuscitation"; Editor of the Journal "Notfall + Rettungsmedizin". He received professional fees for lectures from the following companies: "Forum für medizinische Fortbildung (FomF)", Baxalta Deutschland GmbH, Bayer Vital GmbH, ZOLL Medical Deutschland GmbH, C. R. Bard GmbH, GS Elektromedizinische Geräte G. Stemple $\mathrm{GmbH}$. The other authors do not have a conflict of interest.

\section{Author details}

${ }^{1}$ University of Cologne, Faculty of Medicine and University Hospital of Cologne, Department of Anaesthesiology and Intensive Care Medicine, Kerpener Strasse 62, 50937 Cologne, Germany. 'Emergency Department, University Hospital RWTH Aachen, Pauwelsstrasse 30, 52074 Aachen, Germany. ${ }^{3}$ University of Cologne, Faculty of Medicine, and University Hospital of Cologne, Institute of Medical Statistics and Computational Biology, Kerpener Strasse 62, 50937 Cologne, Germany. ${ }^{4}$ University of Cologne, Faculty of Medicine and University Hospital of Cologne, Centre of Palliative Medicine, Kerpener Strasse 62, 50937 Cologne, Germany.

Received: 26 November 2020 Accepted: 26 July 2021 Published online: 21 August 2021

\section{References}

1. Wong CX, Brown A, Lau DH, Chugh SS, Albert CM, Kalman JM, et al. Epidemiology of sudden cardiac death: global and regional perspectives.
Heart Lung Circ. 2019;28(1):6-14. https://doi.org/10.1016/j.hlc.2018.08.026 Epub 2018/11/30. PubMed PMID: 30482683

2. Sans S, Kesteloot H, Kromhout D. The burden of cardiovascular diseases mortality in Europe. Task force of the European Society of Cardiology on cardiovascular mortality and morbidity statistics in Europe. Eur Heart J. 1997; 18(8):1231-48. Epub 1997/08/01. PubMed PMID: 9458415. https://doi.org/1 0.1093/oxfordjournals.eurheartj.a015434.

3. Hinkelbein J, Böttiger BW. The message is clear to save an additional 100,000 lives per year in Europe: 'harder and faster for cardiopulmonary resuscitation!'. Eur J Anaesthesiol. 2011;28(12):817-8. https://doi.org/10.1097/ EJA.0b013e3283471133 Epub 2011/11/02. PubMed PMID: 22042089.

4. Bobrow BJ, Panczyk M, Subido C. Dispatch-assisted cardiopulmonary resuscitation: the anchor link in the chain of survival. Curr Opin Crit Care. 2012;18(3):228-33. https://doi.org/10.1097/MCC.0b013e328351736b Epub 2012/02/16. PubMed PMID: 22334216.

5. Breckwoldt J, Schloesser S, Arntz HR. Perceptions of collapse and assessment of cardiac arrest by bystanders of out-of-hospital cardiac arrest (OOHCA). Resuscitation. 2009;80(10):1108-13. https://doi.org/10.1016/j. resuscitation.2009.06.028 Epub 2009/07/28. PubMed PMID: 19632755.

6. Gräsner JT, Lefering R, Koster RW, Masterson S, Böttiger BW, Herlitz J, et al. EuReCa ONE-27 nations, ONE Europe, ONE registry: a prospective one month analysis of out-of-hospital cardiac arrest outcomes in 27 countries in Europe. Resuscitation. 2016;105:188-95. https://doi.org/10.1016/j.resuscita tion.2016.06.004 Epub 2016/06/21. PubMed PMID: 27321577.

7. Eisenberg MS, Hallstrom AP, Carter WB, Cummins RO, Bergner L, Pierce J. Emergency CPR instruction via telephone. Am J Public Health. 1985;75(1): 47-50. https://doi.org/10.2105/ajph.75.1.47 Epub 1985/01/01. PubMed PMID: 3966598; PubMed Central PMCID: PMCPMC1646147.

8. Hasselqvist-Ax I, Riva G, Herlitz J, Rosenqvist M, Hollenberg J, Nordberg P, et al. Early cardiopulmonary resuscitation in out-of-hospital cardiac arrest. N Engl J Med. 2015;372(24):2307-15. https://doi.org/10.1056/NEJMoa1405796 Epub 2015/06/11. PubMed PMID: 26061835

9. Sasson C, Rogers MA, Dahl J, Kellermann AL. Predictors of survival from outof-hospital cardiac arrest: a systematic review and meta-analysis. Circ Cardiovasc Qual Outcomes. 2010;3(1):63-81. https://doi.org/10.1161/ CIRCOUTCOMES.109.889576 Epub 2010/02/04. PubMed PMID: 20123673.

10. Tanaka Y, Taniguchi J, Wato Y, Yoshida Y, Inaba H. The continuous quality improvement project for telephone-assisted instruction of cardiopulmonary resuscitation increased the incidence of bystander CPR and improved the outcomes of out-of-hospital cardiac arrests. Resuscitation 2012;83(10):12351241. doi: https://doi.org/10.1016/j.resuscitation.2012.02.013. Epub 2012/03/ 01. PubMed PMID: 22366353.

11. Lin YY, Chiang WC, Hsieh MJ, Sun JT, Chang YC, Ma MH. Quality of audioassisted versus video-assisted dispatcher-instructed bystander cardiopulmonary resuscitation: a systematic review and meta-analysis. Resuscitation. 2018;123:77-85. https://doi.org/10.1016/j.resuscitation.2017.12. 010 Epub 2017/12/16. PubMed PMID: 29242057.

12. Lee DK, Park SM, Kim YJ, Lee CA, Jeong WJ, Kim GW, et al. CPR Guidance by an emergency physician via video call: a simulation study. Emerg Med Int. 2018;2018:1480726. https://doi.org/10.1155/2018/1480726 Epub 2019/01/11. PubMed PMID: 30627442; PubMed Central PMCID: PMCPMC6304577.

13. Ecker $\mathrm{H}$, Wingen $\mathrm{S}$, Hamacher S, Lindacher F, Böttiger BW, Wetsch WA Evaluation of CPR quality via smartphone with a video livestream - a study in a metropolitan area. Prehosp Emerg Care. 2020;25(1):1-6. https://doi. org/10.1080/10903127.2020.1734122. Epub 2020/02/25. PubMed PMID: 32091293.

14. Solomon HV. COVID-19 checklist: Mask, gloves, and video chatting with grandpa. Psychiatry Res. 2020;288:112986. https://doi.org/10.1016/j. psychres.2020.112986 Epub 2020/04/13. PubMed PMID: 32279009; PubMed Central PMCID: PMCPMC7271068.

15. Negro A, Mucci M, Beccaria P, Borghi G, Capocasa T, Cardinali M, et al. Introducing the Video call to facilitate the communication between health care providers and families of patients in the intensive care unit during COVID-19 pandemia. Intensive Crit Care Nurs. 2020:102893. https://doi.org/1 0.1016/j.iccn.2020.102893 Epub 2020/06/25. PubMed PMID: 32576488; PubMed Central PMCID: PMCPMC7247985 competing financial interests or personal relationships that could have appeared to influence the work reported in this paper.

16. Greenhalgh T, Wherton J, Shaw S, Morrison C. Video consultations for covid19. BMJ. 2020;368:m998. https://doi.org/10.1136/bmj.m998 Epub 2020/03/14 PubMed PMID: 32165352. 
17. Ohannessian R, Duong TA, Odone A. Global telemedicine implementation and integration within health systems to fight the COVID-19 pandemic: A call to action. JMIR Public Health Surveill. 2020;6(2):e18810. https://doi.org/1 0.2196/18810 Epub 2020/04/03. PubMed PMID: 32238336; PubMed Central PMCID: PMCPMC7124951.

18. Ecker H, Lindacher F, Adams N, Hamacher S, Wingen S, Schier R, et al. Video-assisted cardiopulmonary resuscitation via smartphone improves quality of resuscitation: a randomised controlled simulation trial. Eur J Anaesthesiol. 2020;37(4):294-302. https://doi.org/10.1097/EJA. 0000000000001177 Epub 2020/02/20. PubMed PMID: 32073408

19. Ecker H, Lindacher F, Dressen J, Wingen S, Hamacher S, Böttiger BW, et al. Accuracy of automatic geolocalization of smartphone location during emergency calls - a pilot study. Resuscitation. 2020;146:5-12. https://doi. org/10.1016/j.resuscitation.2019.10.030 Epub 2019/11/11. PubMed PMID: 31706968.

20. Bolle SR, Scholl J, Gilbert M. Can video mobile phones improve CPR quality when used for dispatcher assistance during simulated cardiac arrest? Acta Anaesthesiol Scand. 2009;53(1):116-20. https://doi.org/10.1111/j.1399-6576.2 008.01779.x Epub 2008/11/27. PubMed PMID: 19032569; PubMed Central PMCID: PMCPMC2659378.

21. Johnsen E, Bolle SR. To see or not to see--better dispatcher-assisted CPR with video-calls? A qualitative study based on simulated trials. Resuscitation. 2008;78(3):320-6. https://doi.org/10.1016/j.resuscitation.2008.04.024 Epub 2008/06/28. PubMed PMID: 18583015.

22. Bolle SR, Johnsen E, Gilbert M. Video calls for dispatcher-assisted cardiopulmonary resuscitation can improve the confidence of lay rescuers-surveys after simulated cardiac arrest. J Telemed Telecare. 2011;17(2):88-92. https://doi.org/10.1258/jtt.2010.100605 Epub 2010/12/09. PubMed PMID: 21139017.

23. Stipulante S, Delfosse AS, Donneau AF, Hartstein G, Haus S, D'Orio V, et al. Interactive videoconferencing versus audio telephone calls for dispatcherassisted cardiopulmonary resuscitation using the ALERT algorithm: a randomized trial. Eur J Emerg Med. 2016;23(6):418-24. https://doi.org/10.1 097/MEJ.0000000000000338.

24. McInnes AD, Sutton RM, Nishisaki A, Niles D, Leffelman J, Boyle L, et al. Ability of code leaders to recall CPR quality errors during the resuscitation of older children and adolescents. Resuscitation. 2012;83(12):1462-6. https:// doi.org/10.1016/j.resuscitation.2012.05.010

25. Al-Rasheed RS, Devine J, Dunbar-Viveiros JA, Jones MS, Dannecker M, Machan JT, et al. Simulation intervention with manikin-based objective metrics improves CPR instructor chest compression performance skills without improvement in chest compression assessment skills. Simul Healthc. 2013;8(4):242-52. https://doi.org/10.1097/SIH.0b013e31828e716d.

26. Hsieh TC, Wolfe H, Sutton R, Myers S, Nadkarni V, Donoghue A. A comparison of video review and feedback device measurement of chest compressions quality during pediatric cardiopulmonary resuscitation. Resuscitation. 2015;93:35-9. https://doi.org/10.1016/j.resuscitation.2015.05. 022 Epub 2015 Jun 4. PMID: 26051808.

27. Cheng A, Overly F, Kessler D, Nadkarni VM, Lin Y, Doan Q, et al. Perception of CPR quality: Influence of CPR feedback, Just-in-Time CPR training and provider role. Resuscitation. 2015;87:44-50. https://doi.org/10.1016/j.resuscita tion.2014.11.015 Epub 2014 Nov 26. PMID: 25433294.

28. Olasveengen TM, Semeraro F, Ristagno G, Castren M, Handley A, Kuzovlev A et al. European resuscitation council guidelines 2021: basic life support. Resuscitation. 2021;161:98-114. https://doi.org/10.1016/j.resuscitation.2021. 02.009.

\section{Publisher's Note}

Springer Nature remains neutral with regard to jurisdictional claims in published maps and institutional affiliations.

Ready to submit your research? Choose BMC and benefit from:

- fast, convenient online submission

- thorough peer review by experienced researchers in your field

- rapid publication on acceptance

- support for research data, including large and complex data types

- gold Open Access which fosters wider collaboration and increased citations

- maximum visibility for your research: over $100 \mathrm{M}$ website views per year

At BMC, research is always in progress.

Learn more biomedcentral.com/submissions 